\title{
Circuit
}

Musiques contemporaines

\section{Introduction : les enjeux structurels de la préservation des technologies dans les arts contemporains}

\section{Guillaume Boutard}

Volume 23, numéro 2, 2013

Préservation du patrimoine culturel contemporain

URI : https://id.erudit.org/iderudit/1018446ar

DOI : https://doi.org/10.7202/1018446ar

Aller au sommaire du numéro

Éditeur(s)

Les Presses de l’Université de Montréal

ISSN

1183-1693 (imprimé)

1488-9692 (numérique)

Découvrir la revue

Citer ce document

Boutard, G. (2013). Introduction : les enjeux structurels de la préservation des technologies dans les arts contemporains. Circuit, 23(2), 5-8.

https://doi.org/10.7202/1018446ar d'utilisation que vous pouvez consulter en ligne. 


\title{
Introduction : les enjeux structurels de la préservation des technologies dans les arts contemporains
}

\author{
Guillaume Boutard
}

Richard [Tuttle] had taught me how to remake them [the string drawings] and that was interesting too because we had hours of a kind of a dance lesson almost, where Richard would show me how to choreograph a certain shape so that I could reproduce it. And what was most interesting about that was that you had to achieve a certain kind of mental state, which was open and almost uncertain, almost well, kind of a meditative state, in order to make the drawings; because otherwise they would come out too perfect [...]. - Marcia Tucker ${ }^{1}$

C'est avec la plus grande précision possible que j’ai évoqué les détails techniques et matériels de l'enregistrement sur bande magnétique car, au cours de ces séances, j'ai appris à quel point la gravure et la restitution du son, et même le jeu du pianiste, sont tributaires de toutes ces conditions. Il s'agissait du premier enregistrement discographique auquel j'assistais personnellement, et je fus effaré de voir cette situation terriblement artificielle, l'ampleur des «impondérables » et des interventions techniques dans la sphère musicale. - Karlheinz Stockhausen ${ }^{2}$

Ces deux citations, prises dans deux contextes très différents qui sont néanmoins ceux que nous présentons pour ce numéro de Circuit, musiques contemporaines, nous montrent l'ampleur de la tâche quand on aborde la problématique de la préservation du patrimoine culturel contemporain dans sa relation avec la technique et la technologie. La question de la transmission évoquée par Marcia Tucker se voit ajouter une problématique, introduite par Karlheinz Stockhausen, qui est celle des interactions au sein de la sphère sociotechnique dont la dimension corollaire dans le domaine des musiques électroacoustiques et mixtes est celle de l'obsolescence technologique et par extension celle de la migration.
1. Scènes bonus de: Chris Maybach (réal.) (2005), Richard Tuttle: Never Not an Artist, DVD, Twelve Films 61391061.

\footnotetext{
2. Dans le livret du disque: Karlheinz Stockhausen (1993), Klavierstücke I-XI / Mikrophonie I \& II, piano Aloys Kontarsky, Sony Classical 01-05334610, p. 22. Texte de Stockhausen (1966-1967), traduction de Françoise Ferlan. Les conditions énumérées par Stockhausen, avec beaucoup d'humour, incluent le dispositif technologique tout autant que le contenu des repas d'Aloys Kontarsky et les rondelles de caoutchouc sous les pieds de la banquette.
} 
3. On citera notamment: Sergio Canazza et Alvise Vidolin (2001), "Introduction: Preserving Electroacoustic Music", Journal of New Music Research, vol. 30, n 4 , p. 289293 ; Joel Chadabe (2001), "Preserving Performances of Electronic Music", Journal of New Music Research, vol. 30, n ${ }^{\circ}$, p. 303-305; Marc Battier (2004), "Electroacoustic Music Studies and the Danger of Loss", Organised Sound, vol. 9, $n^{\circ}$ 1, p. 47-53; Nicola Bernardini et Alvise Vidolin (2005), "Sustainable Live Electroacoustic Music", International Sound and Music Computing Conference, Salerno, Italy.

4. Projet fortement lié à la perspective de Rinehart sur la préservation. Voir: Richard Rinehart (2002), Preserving the Rhizome Artbase, <http://archive. rhizome.org/artbase/preserving-therhizome-artbase-richard-rinehart> (consulté le 6 mai 2013).

5. Projets qui prirent notamment le cas de l'Institut de recherche et coordination acoustique/musique (Ircam) pour leurs études.

6. Musée qu'elle fonda peu après avoir quitté le Whitney Museum, et ceci notamment en raison du scandale lié à l'exposition des œuvres de Richard Tuttle.

7. Notion que l'on trouve aussi chez Rinehart mais encore chez Laurenson. Voir: Richard Rinehart (2004), "A System of Formal Notation for Scoring Works of Digital and Variable Media Art ", Electronic Media Group Annual Meeting of the American Institute for Conservation of Historic and Artistic Works (14 juin), Portland, Oregon, $<$ http://aic.stanford.edu/sg/emg/ library/pdf/rinehart/Rinehart-EMG2004. pdf $>$ (consulté le 6 mai 2013); Pip Laurenson (2006), "Authenticity, Change and Loss in the Conservation of Time-Based Media Installations", Tate Papers, $n^{\circ} 6$, <www.tate.org.uk/ research/publications/tate-papers/ authenticity-change-and-lossconservation-time-based-media>
Depuis le début des années 2000, tout spécialement, de nombreux articles fondateurs ont présenté cette problématique sous différents aspects, que ce soit dans le contexte de la musique électroacoustique, mixte ou de l'électronique en temps réel ${ }^{3}$. Par la suite, plusieurs projets de préservation ont vu le jour, dans le contexte de la production musicale, parmi lesquels on citera le projet Integra qui a regroupé différents centres de recherche, dont le Centre interdisciplinaire de recherche en musique, médias et technologie (CIRMMT) à Montréal, ou dans le cadre des arts des nouveaux médias, comme le projet de Documentation et conservation du patrimoine des arts médiatiques (DOCAM), centré autour de la Fondation Daniel Langlois, ou encore le projet Rhizome ${ }^{4}$. Ce furent aussi des projets liés, de façon plus générale et du point de vue des sciences de l'information, à la préservation des documents numériques qui ont pris comme terrain d'étude le contexte de la production artistique. Ce fut le cas des projets «Cultural, Artistic and Scientific Knowledge for Preservation, Access and Retrieval» (CASPAR) et «International Research on Permanent Authentic Records in Electronic Systems » (InterPARES II) 5 . Il était donc tout indiqué d'illustrer ce numéro avec l'œuvre de Ryoji Ikeda, exposée à la galerie DHC/ART l'année dernière, dont le travail de mise en abîme à travers les données numériques à l'intersection du son et de l'image entre en résonance avec les articles de ce numéro. Dans le texte de présentation de l'inoubliable exposition, John Zeppetelli argumentait ainsi que le flux d'information était à la fois le matériau et le contenu de cette œuvre et concluait que Ryoji Ikeda anéantissait « la documentation cumulative tout en reconstruisant l'édifice de la culture».

Il n'est pas innocent, non plus, que nous citions en exergue la regrettée Marcia Tucker - en dehors d'une admiration partagée pour l'œuvre de Richard Tuttle - puisque c'est elle, en effet, qui fonda le New Museum of Contemporary Art dans la ville de New York ${ }^{6}$, musée qui abrite dorénavant le projet Rhizome. C'est sur cette notion d'interprétation qu'on entrevoit dans le discours de Marcia Tucker, qu'Ariane Noël de Tilly, ancienne collaboratrice du projet DOCAM, élabore son article qui retrace dans un même cadre conceptuel les enjeux de la migration et de l'interprétation ${ }^{7}$ à travers les activités pratiques de l'exposition, de la distribution et de la préservation. À l'heure où plusieurs auteurs ont reproché à la notation de l'électronique d'être encore dans l'enfance de l'art ${ }^{8}$, il est intéressant de voir que le domaine des arts médiatiques, notamment, réinvestit la notion de notation, prescriptive ou descriptive9, et de script comme base de préservation, afin de fournir une base conceptuelle riche de recherches théoriques et pratiques. Ariane Noël de Tilly nous montre les implications de ces notions sur la préservation à tra- 
vers une étude de cas basée sur une œuvre de l'artiste canadien John Massey: As the Hammer Strikes (A Partial Illustration), qui lui permet de mettre en lumière la possibilité de la description, en tant qu'explicitation des médiations qui constituent l'œuvre à travers son parcours, sa généalogie. Comment ne pas voir aussi dans cette étude le parallèle à la problématique de la diffusion de l'électronique dans les musiques électroacoustiques et mixtes dans sa relation au dispositif technologique et à l'acoustique des salles?

Dans un cadre conceptuel convergent, mais un domaine différent, Guillaume Boutard et Fabrice Marandola proposent un projet de préservation qui lui aussi met l'accent sur le cycle de vie des systèmes technologiques à travers les enjeux regroupés de la documentation et de la diffusion du répertoire. Ils prennent donc le parti de considérer les enjeux de la préservation du répertoire à travers une tradition vivante de réutilisation des technologies. La question de la migration recoupe donc celle, fondamentale, de l'intelligibilité des systèmes technologiques pris dans le contexte sociotechnique général de leur utilisation et de la construction des conditions de la médiation technologique. Dans ce cadre, leur projet propose une méthodologie pour faire affleurer le contexte d'émergence des dispositifs technologiques, contexte qui autrement se retrouve enchâssé dans la boîte noire du dispositif déjà cristallisé. Leur approche méthodologique basée sur la sociologie des sciences et des technologies autant que sur les études sur les processus créatifs de, par exemple, Donin et Theureau $(2007)^{10}$, vise à intégrer les méthodes de documentation à l'intérieur des processus créatifs. Leur question est donc aussi celle de la constitution des archives des processus créatifs autant que celle de leur utilisation.

Enfin, si nous gardons l'entrevue passionnante avec André Richard, ancien directeur de l'Experimentalstudio de Fribourg-en-Brisgau (Allemagne), pour la fin de cette introduction au présent numéro, il est cependant difficile de ne pas voir dans ce document (cette archive déjà, sans doute) à la fois une introduction et une conclusion. André Richard y explore et dévoile tour à tour, de façon limpide, tous les grands enjeux de la transmission du répertoire qui sont sporadiquement mis en pratique dans les différents articles de ce numéro. C'est, au-delà de la technologie, l'organisation des compétences, l'évaluation de la migration et, plus fondamentalement encore, la question de la transmission et de l'enseignement, de l'écriture et de la connaissance qui sont à tour de rôle mis en lumière. Quelles sont les conditions de la transmission, quel est l'objet que nous transmettons? Telles sont les questions qui animent André Richard et par là même tout ce numéro de Circuit.

$\mathrm{Si}$ la question centrale de la migration est abordée directement ou en filigrane dans ce numéro, elle n'en reste pas moins l'enjeu technologique
Ainsi Millet déclarait que "dans bien des cas, le conservateur se doit d'être une sorte d'interprète. Lorsque entrent dans la composition des œuvres des objets qui demandent à être renouvelés, ou assemblés différemment en fonction d'un nouvel espace d'exposition, le conservateur, aussi précises que soient les consignes données par l'artiste, devra bien prendre des décisions de sa seule initiative". Catherine Millet (1997), L'art contemporain, Paris, Flammarion, p. 43.

8. Manoury, ironique, écrit que "l'écriture est née en Mésopotamie, environ trois mille ans avant JésusChrist et je vois un parallèle entre la naissance des premières écritures et la situation musicale dans laquelle nous vivons actuellement". Philippe Manoury (1999), "Le stade pictographique de la musique électronique doit être dépassé", in Hugues Vinet et François Delalande (dir.), Interfaces homme-machine et création musicale, Paris, Hermès science publications, p. 205.

Zattra, plus généreuse, déclare: "it could be said that computer score notation is at the same stage that tablature notation was at in the sixteenth and seventeenth centuries". Laura Zattra (2004), "Searching for Lost Data: Outlines of Aesthesic-poietic Analysis", Organised Sound, vol. 9, $n^{\circ} 1$, p. 45.

Pour Bernardini et Vidolin: "live electro-acoustic music currently possesses notational conventions and practices that can be compared at best to middle ages tablatures" (Ioc. cit.).

9. Dans le cadre des définitions proposées par Seeger, bien sûr, mais aussi Kanno. Voir: Charles Seeger (1958), "Prescriptive and Descriptive Music Writing", The Musical Quarterly, vol. 44, no 2, p. 184-195; Mieko Kanno (2007), "Prescriptive Notation: Limits and Challenges", Contemporary Music Review, vol. 26, n² 2, p. 231-254.

10. Nicolas Donin et Jacques Theureau (2007), "Theoretical and Methodological 
Issues Related to Long Term Creative Cognition: The Case of Musical Composition", Cognition, Technology \& Work, vol. 9, n 4, p. 233-251.

11. Philippe Leroux (2011), "... phraser le monde: continuité, geste et énergie dans l'œuvre musicale", Circuit, musiques contemporaines, vol. 21, $\mathrm{n}^{\circ} 2$, p. 41. majeur. Migration, processus créatifs et constitution des archives ne sont bien que différentes faces de cette problématique de la préservation du patrimoine culturel contemporain. Deux points de vue s'établissent à travers une question inversée: la préservation passe-t-elle par la transmission, approche présentée de façon récurrente dans ce numéro, ou est-ce la transmission qui passe par la préservation, approche liée à des projets comme Integra. Si la première position tend à favoriser des méthodes de recherche qualitatives, la seconde, elle, s'est concentrée, au travers des projets de recherche et développement montés ces dernières années, sur des questions quantitatives liées à l'ingénierie. Ces projets visent à cadrer la technologie dans une utilisation raisonnée et, en ce sens, répondent à l'inquiétude de Philippe Leroux qui déclarait, dans un précédent numéro de Circuit (2011), qu’ «il est instamment nécessaire pour la sensibilité contemporaine de comprendre comment utiliser au mieux ces créations [outils audio-vidéo] dont elle n'est pas directement responsable, et de stopper ou du moins de ralentir, au moins momentanément, leur prolifération ${ }^{11}$ ».

Ce numéro montre que c'est, sans doute, dans l'articulation entre, d'un côté, des projets comme ceux qui visent une relation technocentrique à la migration - que les stratégies soient celles de la réduction ou de la formalisation - et, d'un autre côté, les recherches et les projets sociotechniques qui marquent ce numéro, que l'on pourra penser les futurs cadres de préservation. On peut donc dire que la question posée comme causalité dans les deux approches est sans doute mal formulée dans la mesure où nous avons bien affaire à un dialogue entre transmission et préservation des agencements, plutôt qu'à une exclusion ou à des processus parallèles qui se confirmeraient ou s'infirmeraient. Ce numéro de Circuit met en avant la conviction que nous sommes sans doute à l'aube d'une nouvelle génération de systèmes théoriques et pratiques de préservation du patrimoine culturel contemporain.

Le dossier thématique est complété par un Cahier d'analyse rédigé par Maxime McKinley qui explore l'œuvre This Will Not Be Televised (20052007) de la compositrice Nicole Lizée, originaire de la Saskatchewan. S'ensuivent les traditionnelles Actualités de Circuit. La rubrique comprend deux comptes rendus de Robert Hasegawa et Éric Legendre, le premier sur le récent ouvrage dirigé par Louise Duchesneau et Wolfgang Marx sur György Ligeti (2011), le second sur La Société de musique contemporaine du Québec (2011) de Réjean Beaucage. Fidèle à ses habitudes, la revue est agrémentée des Nouveautés en bref, compte rendu rédigé par Cléo Palacio-Quintin. 